\title{
Monotone Data Samples Do Not Always Generate Monotone Fuzzy If-Then Rules
}

\author{
Chin Ying Teh and Kai Meng Tay
}

\begin{abstract}
The Wang-Mendel (WM) method is one of the earliest methods to learn fuzzy If-Then rules from data. In this article, the WM method is used to generate fuzzy If-Then rules for a zero-order Takagi-Sugeno-Kang (TSK) fuzzy inference system (FIS) from a set of multi-attribute monotone data. Convex and normal trapezoid fuzzy sets are used as fuzzy membership functions. Besides that, a strong fuzzy partition strategy is used. Our empirical analysis shows that a set of multi-attribute monotone data may lead to non-monotone fuzzy If-Then rules. The same observation can be made, empirically, using adaptive neuro-fuzzy inference system (ANFIS), a well-known and popular FIS model with neural learning capability. This finding is important for the modeling of a monotone FIS model, because it shows that even with a "clean" data set pertaining to a monotone system, the generated fuzzy If-Then rules may need to be preprocessed, before being used for FIS modeling. In short, it is imperative to develop methods for preprocessing non-monotone fuzzy rules from data, e.g., monotone fuzzy rules relabeling, or removing non-monotone fuzzy rules, is important (and is potentially necessary) during the course of developing data-driven FIS models.
\end{abstract}

Keywords Fuzzy If-Then rules - The Wang-Mendel method • ANFIS • Monotonicity property - Multi-attribute monotone data - Monotone fuzzy rule relabeling • Interval-valued fuzzy rules

\section{Introduction}

Fuzzy inference systems (FISs) with fuzzy If-Then rules have been successfully applied to many real-life applications $[1,2]$. Traditionally, fuzzy If-Then rules are obtained from human experts. Owing to the difficulty in soliciting fuzzy rules from

C.Y. Teh · K.M. Tay $(\bowtie)$

Faculty of Engineering, Universiti Malaysia Sarawak, Kota Samarahan, Malaysia

e-mail: kmtay@feng.unimas.my 